Jean Bourgain · Gady Kozma

\title{
One cannot hear the winding number
}

Received December 15, 2005 and in revised form November 21, 2006

Abstract. We construct an example of two continuous maps $f$ and $g$ of the circle to itself with $|\widehat{f}(n)|=|\widehat{g}(n)|$ for all $n \in \mathbb{Z}$ but with different winding numbers, answering a question of Brezis.

Keywords. Winding number, Fourier coefficients, Pauli partners, random Fourier series

\section{Introduction}

A continuous cycle $f$ in $\mathbb{C} \backslash\{0\}$ has a well defined winding number around zero, which we shall denote by wind $f$. If $f$ is smooth and its image is in the circle $\mathbb{T}=\{z:|z|=1\}$ then the winding number has an elegant formula using the Fourier coefficients of $f$ (we consider $f$ as a function from $[0,1]$ to $\mathbb{T}$ ). Indeed, by the residue formula for the function $z^{-1}$ we have

$$
\text { wind } f=\frac{1}{2 \pi i} \int \frac{f^{\prime}}{f}
$$

and since $1 / f=\bar{f}$ we get

$$
\text { wind } f=\frac{1}{2 \pi i} \int f^{\prime} \bar{f}=\frac{1}{2 \pi i} \sum \widehat{f^{\prime}}(n) \widehat{\widehat{f}(n)}=\sum n|\widehat{f}(n)|^{2} .
$$

This paper is part of a line of research trying to understand what role smoothness plays in (1). See Brezis [B06] for a fascinating review of related results and problems, high dimensional analogs and applications.

This material is based upon work supported by the National Science Foundation under agreement DMS-0111298. Any opinions, findings and conclusions or recommendations expressed in this material are those of the authors and do not necessarily reflect the views of the National Science Foundation.

J. Bourgain: Institute for Advanced Study, 1 Einstein Dr., Princeton, NJ 08540, USA; e-mail: bourgain@ias.edu

G. Kozma: The Weizmann Institute of Science, Rehovot 76100, Israel; e-mail: gady.kozma@weizmann.ac.il

Mathematics Subject Classification (2000): Primary 42A16; Secondary 42A61, 55M25 
The earliest investigations in this direction are due to L. Boutet de Monvel and O. Gabber. They realized that the left-most equality in (1) makes sense for functions in the fractional Sobolev space $W^{1 / 2,2}$, when the integral is understood in the sense of $W^{1 / 2,2}$ $W^{-1 / 2,2}$ duality (see the appendix of [BGP91]). This equality allowed them to extend the notion of winding number to the discontinuous part of $W^{1 / 2,2}$. By approximating with continuous functions they showed that this generalized winding number was still an integer. Interestingly, $W^{1 / 2,2}$ is exactly the space of functions for which the right hand side of (1) converges absolutely, but apparently Boutet de Monvel and Gabber were not aware of (1). The connection to Fourier expansion was discovered by Brezis in 1995 (following a question of Gelfand) and immediately begot new questions.

As a side remark, defining the winding number using approximation by continuous functions is most natural in the space VMO of functions of vanishing mean oscillation (VMO is the closure of the continuous functions in BMO and, heuristically, it relates to BMO like continuous functions relate to $L^{\infty}$ ). Note that VMO contains $W^{1 / 2,2}$ and the VMO-winding number agrees with both the definition of [BGP91] and with $\left[1\right.$ ) on $W^{1 / 2,2}$ (see [B06]). A high dimensional analog, the VMO-degree, was developed by Brezis and Nirenberg [BN95] (see also [BC83]).

Returning to 11 , when one leaves $W^{1 / 2,2}$ the picture gets more complicated. Following a question of Brezis, Korevaar [K99] showed that in general the sum [1], considered as the balanced limit

$$
\lim _{N \rightarrow \infty} \sum_{n=-N}^{N} n|\widehat{f}(n)|^{2},
$$

may converge to any desired value different from the winding number, including $\pm \infty$, or may diverge. Replacing convergence with Abel summability does not change the picture. On the other hand, Korevaar shows that (1) does hold for continuous functions with bounded variation (this class is not contained in $W^{1 / 2,2}$ ). More intriguing, perhaps, is Kahane's proof [K05] that for Hölder functions with exponent $>1 / 3$ one can retrieve the winding number by summing (1) using Riemann's summation method. Brezis [B06, Theorem 5] then noticed that Kahane's proof works in the space $W^{1 / 3,3}$. In particular Kahane's argument shows that in $W^{1 / 3,3}$ the absolute values of the Fourier transform determine the winding number, which can be picturesquely described as "hearing the winding number". It is conjectured that one can hear the winding number in the class $W^{1 / p, p}$ for any $p$. Here $W^{1 / p, p}$ is in the sense of Sobolev-Slobodetskiĭ spaces (see e.g. [KJF77]). We remind the reader that in this case the definition of these spaces simplifies to the condition

$$
W^{1 / p, p}=\left\{f: \iint \frac{|f(x)-f(y)|^{p}}{|x-y|^{2}}<\infty\right\} .
$$

This is an increasing family of classes and all $W^{1 / p, p} \subset \mathrm{VMO}$.

Korevaar's negative result, while strongly hinting that one cannot hear the winding number in general, does not actually preclude it. In this paper we show that some kind of smoothness must be assumed in order to hear the winding number. Specifically we show

Theorem. There exist two continuous functions $f, g: \mathbb{T} \rightarrow \mathbb{T}$ with $|\widehat{f}(n)|=|\widehat{g}(n)|$ for all $n$ and with different winding numbers. 
The constructed $f$ and $g$ are highly singular. We made no attempt to optimize the construction in this respect nor do we believe that our techniques are adequate for this purpose. In fact, even forcing $f$ and $g$ to be continuous, as opposed to just VMO, required a non-negligible increase in the complexity of the proof. Another interesting question which we cannot address at this time is whether one can strengthen $|\widehat{f}(n)|=|\widehat{g}(n)|$ to $\widehat{f}(n)= \pm \widehat{g}(n)$.

We remark that functions with identical absolute value and identical absolute value of the Fourier transform are called Pauli partners. Thus in this language the theorem states that there exist two Pauli partners $f$ and $g$ with $|f|=1$ and different winding numbers. For many interesting techniques for producing Pauli partners, see [J99].

\subsection{About the proof}

Inspired by de Leeuw-Kahane-Katznelson [dLKK77] we construct $f$ and $g$ by an iterative correction scheme with each stage combining a deterministic step and a probabilistic step. We start with functions which take values outside $\mathbb{T}$, so (1) does not hold and one may construct trigonometric polynomials with $|\widehat{f}(n)|=|\widehat{g}(n)|$ and different winding numbers quite easily (see page 656). At each stage the polynomials are extended so as to continue satisfying $|\widehat{f}(n)|=|\widehat{g}(n)|$ and preserve their winding number while getting closer and closer to $\mathbb{T}$.

Let us describe one correction. Assume $|f|$ is too low in an interval $I$. We always correct upwards, increasing $|f|$ - this is a corollary of the fact that extending a polynomial increases its $L^{2}$ norm-so our lemmas are usually formulated non-symmetrically with respect to $|f|$. We wish to correct $f$ on $I$ (call the correction $F$ ) in such a way that $F$ has bigger absolute value on $I$ but is highly oscillatory so that $F-f$ lives essentially in the high end of the spectrum.

We employ two different techniques to get $F$ from $f$. The first, and simpler, is described in Lemma 2. Its advantage is that $F$ has the desired properties on all of $I$. Its disadvantage is that $F-f$ has no structure that can be used in order to extend the other polynomial, $g$. Hence when using this technique we simply define

$$
G=g+\sum \pm \widehat{F-f}(n) e^{i n t}
$$

where \pm are random signs. If $I$ is sufficiently small then $\|F-f\|_{2}$ would be small, and we could bound $\|G-g\|_{\infty}$ efficiently by known properties of random trigonometric series.

The second technique is used in Lemma 5 and especially in Lemma 7. We construct $F-f$ so as to have many small pieces sitting in different areas of the spectrum and use this structure in order to construct $G$ so that its distance from a unimodular function is decreased as little as possible. The disadvantage of this technique is that we have not figured out how to correct on the whole of $I . F$ has the desired absolute value on most of $I$ but a small exceptional set remains, and is handled using the first technique. 


\subsection{Notations}

We shall consider functions from $[0,1]$ into $\mathbb{C}$, which we consider as periodic e.g. when we say that $f$ is continuous we mean also that $f(0)=f(1)$. A rotation is the action of transforming $f(t)$ into $f(t+a)$ for some $a \in[0,1]$, again periodically. The Fourier coefficients are defined by $\widehat{f}(n)=\int_{0}^{1} f(t) e^{-2 \pi i n t}$. For a trigonometric polynomial $f$ we define its spectrum, denoted by spec $f$, and its degree, denoted by $\operatorname{deg} f$, by

$$
\operatorname{spec} f:=\{n \in \mathbb{Z}: \widehat{f}(n) \neq 0\}, \quad \operatorname{deg} f:=\max \{|n|: n \in \operatorname{spec} f\} .
$$

If $f$ and $F$ are two trigonometric polynomials we say that $F$ extends $f$ if they are identical on the hull of the spectrum of $f$, that is,

$$
\widehat{F}(n)=\widehat{f}(n) \quad \forall|n| \leq \operatorname{deg} f .
$$

$\omega(\delta ; f)$ will denote the modulus of continuity, namely

$$
\omega(\delta ; f):=\max _{|x-y| \leq \delta}|f(x)-f(y)| .
$$

We shall denote by $C^{n}, n \in \mathbb{N}$, the space of functions with $n$ continuous derivatives and by $\left.C^{\alpha}, \alpha \in\right] 0,1[$, the space of Hölder continuous functions of order $\alpha$. The respective norms are

$$
\|f\|_{C^{n}}:=\max \left\{\|f\|_{\infty},\left\|f^{(n)}\right\|_{\infty}\right\}, \quad\|f\|_{C^{\alpha}}:=\max _{x \neq y} \frac{|f(x)-f(y)|}{|x-y|^{\alpha}}
$$

(we shall not need other $C^{x} \mathrm{~s}$-in fact we shall only use $C^{2}, C^{1}$ and $C^{1 / 2}$ ). Other norms we will use are

$$
\|f\|_{2}:=\sqrt{\int_{0}^{1}|f|^{2}}, \quad\|f\|_{\infty}:=\underset{t \in[0,1]}{\operatorname{ess} \sup }|f(t)|,
$$

and we denote by $V(f)$ the total variation of $f$. We also remind the reader that

$$
V(f g) \leq V(f)\|g\|_{\infty}+V(g)\|f\|_{\infty}, \quad\|f g\|_{C^{\alpha}} \leq\|f\|_{C^{\alpha}}\|g\|_{\infty}+\|g\|_{C^{\alpha}}\|f\|_{\infty} .
$$

By $f_{+}$we mean $\max \{f, 0\}$. For a set $E$ we denote by $\mathbf{1}_{E}$ the indicator function which is 1 on $E$ and 0 elsewhere. $\lfloor x\rfloor$ and $\lceil x\rceil$ denote, respectively the floor and ceiling functions, i.e. the largest integer $\leq x$ and the smallest integer $\geq x$. By $C$ and $c$ we shall denote absolute constants whose precise value is unimportant as far as this paper is concerned, and could change from formula to formula or even within the same formula. $C$ will pertain to constants which are "big enough" and $c$ to constants which are "small enough". We will number a few $C$ and $c s-$ only those which we will reference later on. When we say " $x$ is sufficiently large" we mean " $x>C$ ". 


\section{Proof}

\subsection{The local correction scheme}

Lemma 1. Let $h$ be a $C^{2}$ function. Then

$$
V\left(\sqrt{h_{+}}\right) \leq \sqrt{\|h\|_{C^{2}}}
$$

Proof. In fact we will prove the stronger

$$
V(\sqrt{|h|}) \leq \sqrt{\|h\|_{C^{2}}}
$$

Let $I=[a, b]$ be an interval such that $h^{\prime}(a)=h^{\prime}(b)=0$ and $h$ is monotone on $[a, b]$. Then

$$
V_{I}(h)=|h(b)-h(a)| \leq(b-a) \max _{t \in I}\left|h^{\prime}(t)\right| \leq \frac{1}{2}(b-a)^{2} \max _{t \in I}\left|h^{\prime \prime}(t)\right|
$$

where $V_{I}(h)$ is the variation of $h$ on $I$. If in addition $h(t) \neq 0$ for all $\left.t \in\right] a, b[$ then

$$
V_{I}(\sqrt{|h|})=|\sqrt{|h(b)|}-\sqrt{|h(a)|}| \leq \sqrt{|h(b)-h(a)|} \leq \sqrt{\frac{1}{2}\|h\|_{C^{2}}}(b-a) .
$$

If $h(t)=0$ for some $t \in] a, b[$ then

$$
V_{I}(\sqrt{|h|})=\sqrt{|h(b)|}+\sqrt{|h(a)|} \leq \sqrt{2|h(b)-h(a)|}
$$

so in both cases

$$
V_{I}(\sqrt{|h|}) \leq \sqrt{\|h\|_{C^{2}}}(b-a) .
$$

A similar argument shows that if $h^{\prime}(t)=0$ for any $t \in[a, b]$ (and without assuming monotonicity of $h$ ) then

$$
|\sqrt{|h(a)|}-\sqrt{|h(b)|}| \leq \sqrt{\|h\|_{C^{2}}}(b-a) .
$$

Let now $a_{1}<\cdots<a_{N} \in[0,1]$. We need to estimate the variation with respect to $a_{1}, \ldots, a_{N}$. Clearly we may add points and we add, for any segment $\left[a_{i}, a_{i+1}\right]$ where $h$ is not monotonic, the maximal and minimal points in $\left[a_{i}, a_{i+1}\right]$ where $h^{\prime}=0$, removing duplicates if they arise (each may be equal to the boundary point, and the two points may be equal). Denote the new list also by $a_{i}$. Let $i_{1}<\cdots<i_{K}$ be the points where $h^{\prime}\left(a_{i_{k}}\right)=0$ and assume that $a_{i_{1}}=0$ and $a_{i_{K}}=1$ (as we may, by rotating $h$ and the $a_{i} \mathrm{~s}$ and adding one $a_{i}$, if necessary). It is now easy to verify that

$$
h \text { is monotone on }\left[a_{i_{k}}, a_{i_{k+1}}\right] \text { whenever } i_{k+1}>i_{k}+1 \text {. }
$$

Let us now write

$$
V\left(\sqrt{|h|} ; a_{1}, \ldots, a_{N}\right):=\sum_{i=1}^{N-1}\left|\sqrt{\left|h\left(a_{i+1}\right)\right|}-\sqrt{\left|h\left(a_{i}\right)\right|}\right|=\sum_{k=1}^{K-1} v_{k}
$$


where

$$
v_{k}:=\sum_{i=i_{k}}^{i_{k+1}-1} \mid \sqrt{\left|h\left(a_{i+1}\right)\right|}-\sqrt{\left|h\left(a_{i}\right)\right|} .
$$

However, by (5) we can use (3) for $h$ monotone on $\left[a_{i_{k}}, a_{i_{k+1}}\right]$ and $(4)$ for $i_{k+1}=i_{k}+1$, and in both cases we get $v_{k} \leq \sqrt{\|h\|_{C^{2}}}\left(a_{i_{k+1}}-a_{i_{k}}\right)$. This proves the lemma.

Lemma 2. Let $f$ be a trigonometric polynomial with $1-c_{1}<|f|<1+c_{1}$ for some absolute constant $0<c_{1}<1$. Let $[a, b] \subset \mathbb{T}$ be some interval such that $|f(a)|=|f(b)|$ and $|f(t)|<|f(a)|$ for all $t \in[a, b]$. Let $\epsilon \in] 0,1[$ be some parameter. Then one can extend $f$ as $F$ such that

$$
\begin{aligned}
& || F(t)|-| f(a) \|<\epsilon \quad \forall t \in[a, b], \\
& |F(t)-f(t)|<\epsilon \quad \forall t \notin[a, b], \\
& \operatorname{deg} F \leq C(\operatorname{deg} f)^{12} / \epsilon^{4} .
\end{aligned}
$$

Proof. Assume $a=0$ (otherwise we can rotate the whole thing). We shall need the following function, defined on $[0, \infty[$ :

$$
\varphi(x)=\sum_{j=1}^{\infty} \frac{(-1)^{j+1}}{(2 j) !}\left(\begin{array}{c}
2 j \\
j
\end{array}\right) 4^{-j} x^{j} .
$$

Clearly this is an analytic function with $\varphi(0)=0$ and $\varphi^{\prime}(0)>0$. Hence we may invert it in some neighborhood of 0 . We get an analytic monotone function $\psi:[0, c] \rightarrow[0,1]$ with $\psi^{\prime} \leq C$ and $\psi^{\prime \prime} \leq C$.

The construction now goes as follows. Write $\tau=|f(0)|, N=\operatorname{deg} f$ and let $M=$ $M(N)$ be some number to be fixed later. Define

$$
\begin{aligned}
\delta(t) & = \begin{cases}\sqrt{\psi(1-|f(t)| / \tau)}, & t \in[0, b], \\
0, & \text { otherwise, }\end{cases} \\
f_{2}(t) & = \begin{cases}\frac{f(t)}{|f(t)|} \tau e^{i \delta(t) \sin M t}, & t \in[0, b], \\
f(t), & \text { otherwise. }\end{cases}
\end{aligned}
$$

If $c_{1}$ is sufficiently small then $\delta$ is always well defined. Fix some value of $c_{1}<1 / 2$ satisfying this requirement. The following properties of $f_{2}$ now follow:

$$
\begin{array}{rlrl}
\left|f_{2}(t)\right| & =\tau & & \forall t \in[0, b], \\
f_{2}(t) & =f(t) & \forall t \notin[0, b] .
\end{array}
$$

To estimate $\widehat{f_{2}}$ develop $e^{i \delta(t) \sin M t}$ in a Taylor series. We get

$$
e^{i \delta(t) \sin M t}=1+\sum_{j=1}^{\infty} \frac{1}{j !}(i \delta(t) \sin M t)^{j} .
$$


Expanding $\sin ^{j} M t=\left(\left(e^{i M t}-e^{-i M t}\right) / 2 i\right)^{j}$ we get for odd $j$ a sum with no constant coefficient and for even $j$ the constant coefficient is $\left(\begin{array}{c}j \\ j / 2\end{array}\right) 2^{-j}$. Hence we may write, for all $t \in[0, b]$,

$$
\begin{aligned}
e^{i \delta(t) \sin M t} & =1+\sum_{j=1}^{\infty} \frac{(-1)^{j}}{(2 j) !}\left(\begin{array}{c}
2 j \\
j
\end{array}\right) 4^{-j} \delta^{2 j}(t)+R(t)=1-\varphi\left(\delta^{2}(t)\right)+R(t) \\
& =\frac{|f(t)|}{\tau}+R(t)
\end{aligned}
$$

where $R(t)$ contains all terms depending on $M$. Hence we get $f_{2}(t)=f(t)+S(t)$ where

$$
S(t):= \begin{cases}\frac{f(t)}{|f(t)|} \tau R(t), & t \in[0, b], \\ 0, & \text { otherwise. }\end{cases}
$$

The next step is to ask how large $M$ has to be to ensure that $S$ lives only in the high end of the spectrum. This is pretty straightforward, but let us do it in detail nonetheless.

Let therefore $|n| \leq N$ and let us examine $\widehat{S}(n)$. Integrating by parts we get

$$
\widehat{S}(n)=\int_{0}^{1} S(t) e^{-2 \pi i n t} d t=\int_{0}^{1} S(t) d t+2 \pi i n \int_{0}^{1} e^{-2 \pi i n s} \int_{0}^{s} S(t) d t d s .
$$

To estimate this we start by writing, for $s \in[0, b]$,

$$
\left|\int_{0}^{s} S(t) d t\right| \leq \sum_{j=1}^{\infty} \frac{1}{j !} \sum_{k=0}^{j}\left(\begin{array}{l}
j \\
k
\end{array}\right) 2^{-j} \tau\left|\int_{0}^{s} e^{i M(j-2 k) t} \frac{f(t)}{|f(t)|} \delta^{j}(t) d t\right|
$$

where $\Sigma^{\prime}$ means that for $j$ even the sum does not contain the term $k=j / 2$. Every term on the right is again estimated by integration by parts to get

$$
\left|\int_{0}^{s} e^{i M(j-2 k) t} \frac{f(t)}{|f(t)|} \delta^{j}(t) d t\right| \leq \frac{2}{M}\left(C+V\left(\frac{f}{|f|} \delta^{j}\right)\right) .
$$

For $j$ even we can simply estimate the variation by the maximum of the derivative. We use Bernstein's inequality 1 to get $\left|f^{\prime}(t)\right| \leq N\|f\|_{\infty} \leq N\left(1+c_{1}\right)$ and $\left|(|f|)^{\prime}(t)\right| \leq\left|f^{\prime}(t)\right| \leq$ $C N$. Further,

$$
\left|\left(\delta^{2 j}\right)^{\prime}\right| \leq \frac{C j}{\tau}|f|^{\prime} \leq C j N
$$

and therefore

$$
V\left((f /|f|) \delta^{2 j}\right) \leq\left\|(f /|f|) \delta^{2 j}\right\|_{C^{1}} \leq C j N
$$

\footnotetext{
1 In fact here it is enough to use the trivial inequality $\left|f^{\prime}\right| \leq N^{2}\|f\|_{\infty}$.
} 
In the case of $j$ odd we use Lemma 1 to get

$$
\begin{gathered}
V\left(\frac{f}{|f|} \delta^{2 j+1}\right) \stackrel{\text { 2 }}{\leq} C V\left(\frac{f}{|f|} \delta^{2 j}\right)+C V(\delta) \leq C j N+\sqrt{\left\|\psi\left(1-\frac{|f|}{\tau}\right)\right\|_{C^{2}}} \\
\\
\stackrel{(*)}{\leq} C j N+C \sqrt{\||f|\|_{C^{2}}+\||f|\|_{C^{1}}^{2}} \leq C j N
\end{gathered}
$$

where in $(*)$ we used the fact that $\psi^{\prime} \leq C$ and $\psi^{\prime \prime} \leq C$, and in the last inequality we again used Bernstein's inequality. Inserting this into (12) and the result into (11) and summing over $k$ and $j$ gives $\left|\int_{0}^{s} S(t)\right| d t \leq C N / M$. Hence $|\widehat{S}(n)| \leq C n N / M$. Summing over $n$ we get

$$
\left\|\sum_{n=-N}^{N} \widehat{S}(n) e^{2 \pi i n t}\right\|_{\infty} \leq \frac{C N^{3}}{M} .
$$

Next we need to estimate $|\widehat{S}(n)|$ for large $n$. The square root in the definition of $\delta$ means $S$ is not smooth, but we shall show that $S$ is Hölder $-\frac{1}{2}$. We remind the reader that in general such functions have uniformly convergent Fourier expansion. In fact, by the Dini-Lipschitz test [Z68, §2.71]

$$
\left\|\sum_{|n|>v} \widehat{S}(n) e^{i n t}\right\|_{\infty} \leq C v^{-1 / 2}(\log v)\|S\|_{C^{1 / 2}} .
$$

Write therefore

$$
\begin{aligned}
\left\|e^{i M(j-2 k) t} \frac{f}{|f|} \delta^{j}\right\|_{C^{1 / 2}} & \stackrel{2}{\underline{2}}\left\|\frac{f}{|f|} \delta^{j}\right\|_{C^{1 / 2}}+C\left\|e^{i M(j-2 k) t}\right\|_{C^{1 / 2}} \\
& \leq\left\|\frac{f}{|f|} \delta^{j}\right\|_{C^{1 / 2}}+C \sqrt{j M} .
\end{aligned}
$$

To estimate the term $(f /|f|) \delta^{j}$, note that for even $j$ we have

$$
\left\|\frac{f}{|f|} \delta^{2 j}\right\|_{C^{1 / 2}} \leq\left\|\frac{f}{|f|} \delta^{2 j}\right\|_{C^{1}} \stackrel{13}{\leq} C j N .
$$

Further, since

$$
\|\delta\|_{C^{1 / 2}} \leq \sqrt{\left\|\psi\left(1-\frac{|f|}{\tau}\right)\right\|_{1}} \leq C
$$

we see that (17) holds for $2 j+1$ as well. Hence

$$
\left\|e^{i M(j-2 k) t} \frac{f}{|f|} \delta^{j}\right\|_{C^{1 / 2}} \leq C j N+C \sqrt{j M} .
$$

We now sum over $k$ and $j$ with the final result being $\|S\|_{C^{1 / 2}} \leq C(N+\sqrt{M})$. Returning to 16 this gives

$$
\left\|\sum_{|n|>v} \widehat{S}(n) e^{i n t}\right\|_{\infty} \leq C v^{-1 / 2}(\log v)(N+\sqrt{M}) .
$$


Combining (15) and [18) allows us to define

$$
F=f+\sum_{N<|n| \leq M^{4}} \widehat{S}(n) e^{i n t}
$$

and get $\left\|F-f_{2}\right\|_{\infty} \leq C N^{3} / M$. We pick $M=\left\lceil C N^{3} / \epsilon\right\rceil$, get

$$
\left\|F-f_{2}\right\|_{\infty} \leq \epsilon
$$

and the lemma is proved (remember 97 and $(10)$ ).

Lemma 3. With the notations of Lemma 2, if one replaces $[a, b]$ with a simple set $E$ (such that $|f|$ is constant on $\partial E$ ) then the lemma holds with condition (8) replaced by

$$
\operatorname{deg} F \leq C(\operatorname{deg} f)^{16} / \epsilon^{4} .
$$

Proof. We note that $|f|^{2}$ is a trigonometric polynomial of degree $\leq 2 \operatorname{deg} f$ and hence the number of solutions of $|f|^{2}=\tau$ for any number $\tau$ is $\leq 4 \operatorname{deg} f+1$ (we assume here that $|f|$ is not constant-if it is, just take $F=f$ ). Therefore $E$ is composed of no more than $2 \operatorname{deg} f$ intervals $I_{k}$. Apply Lemma 2 for each interval $I_{k}$ with $\epsilon_{\mathrm{Lemma} 2}=\epsilon / 2 \operatorname{deg} f$. Call the resulting functions $F\left(I_{k}\right)$ and define $F=f+\sum_{k}\left(F\left(I_{k}\right)-f\right)$. All the conditions are obviously satisfied.

Lemma 4. Let $\eta \in \mathbb{C}$ and $\tau \in \mathbb{R}^{+}$with $|\eta|<\tau$. Then

$$
\left|\eta \pm \sqrt{\tau^{2}-|\eta|^{2}} \frac{i \eta}{|\eta|}\right|=\tau,
$$

and for any $\sigma \in[-1,1]$,

$$
|\eta| \leq\left|\eta+\sigma \sqrt{\tau^{2}-|\eta|^{2}} \frac{i \eta}{|\eta|}\right| \leq \tau .
$$

This follows from Pythagoras' theorem since we are adding orthogonal vectors.

Lemma 5. Let $f, \epsilon$ and $[a, b]$ be as in Lemma 2 and let $\left[a^{\prime}, b^{\prime}\right]$ be another interval, with $b^{\prime}-a^{\prime} \geq b-a$. Let $g$ be a polynomial satisfying $|\widehat{f}(n)|=|\widehat{g}(n)|$ for all $n$. Then one can extend $f$ and $g$ as $F$ and $G$ still satisfying $|\widehat{F}(n)|=|\widehat{G}(n)|$ and such that (6), (7) hold, as well as

$$
\begin{array}{lll}
|g(t)-G(t)|<C \sqrt{(b-a) /\left(b^{\prime}-a^{\prime}\right)} & \forall t \in\left[a^{\prime}, b^{\prime}\right], \\
|g(t)-G(t)|<\epsilon & \forall t \notin\left[a^{\prime}, b^{\prime}\right] .
\end{array}
$$

Proof. Assume without loss of generality that $a=a^{\prime}=0$. Let $l$ satisfy that $4^{-l} \geq b / b^{\prime}>$ $4^{-l-1}$, and assume $l>0$ (otherwise one can take $F$ from Lemma 2 and $G=g+F-f$ ). Let $\delta \in] 0, \epsilon$ [ and $M$ be some parameters to be fixed later- $\delta$ will be taken sufficiently small and $M$ sufficiently large, depending on $\delta$. Let $\psi$ be an $M$-approximation of the first Rademacher function $r_{1}:=\mathbf{1}_{[0,1 / 2]}-\mathbf{1}_{[1 / 2,1]}$, namely

$$
\psi=\sum_{|n| \leq M} \widehat{r}_{1}(n) \frac{M-|n|}{M} e^{i n t} .
$$


As is well known, $\psi$ is real, $|\psi| \leq 1$ and

$$
\left|r_{1}(t)-\psi(t)\right| \leq C M^{-1 / 2} \quad \forall t,\langle 2 t\rangle>M^{-1 / 2}
$$

where $\langle x\rangle$ is defined (somewhat nonstandardly) as the distance of $x$ from the integers. For any integer $m$ define $\psi_{[m]}$ using

$$
\psi_{[m]}(t)=\psi(m t)
$$

where we understand $\psi$ as a 1-periodic function. We define $3 l$ functions which mimic the behavior of $3 l$ different Rademacher functions:

$$
s_{i}:=\psi_{\left[(3 M)^{i}\right]}, \quad i=1, \ldots, 3 l .
$$

The reason we are approximating the Rademacher functions is the following innocuous equality:

$$
\left|r_{1}+r_{2}+r_{3}-r_{1} r_{2} r_{3}\right|=2,
$$

which holds at every point except the jump points. This of course holds for other triplets, i.e. $\left|r_{3 i-2}+r_{3 i-1}+r_{3 i}-r_{3 i-2} r_{3 i-1} r_{3 i}\right|=2$. With this in mind let us construct $4^{l}$ functions which mimic products of the four Walsh functions $r_{1}, r_{2}, r_{3},-r_{1} r_{2} r_{3}$. Formally, for every sequence $\left\{\epsilon_{i}\right\}_{i=1}^{l}$ with $\epsilon_{i} \in\{1,2,3,4\}$ we define

$$
\sigma_{\left\{\epsilon_{i}\right\}}=2^{-l} \prod_{i=1}^{l} \begin{cases}s_{3(i-1)+\epsilon_{i}}, & \epsilon_{i}=1,2,3, \\ -s_{3 i-2} s_{3 i-1} s_{3 i}, & \epsilon_{i}=4 .\end{cases}
$$

For convenience, if $j=\sum_{i=1}^{\alpha}\left(\epsilon_{i}-1\right) 4^{i-1}$ is some number between 0 and $4^{l}-1$ we will write $\sigma_{j}:=\sigma_{\left\{\epsilon_{i}\right\}}$. The $\sigma_{j}$ s have the following properties:

(i) For all $j$ and $t$,

$$
\left|\sigma_{j}(t)\right| \leq 2^{-l}
$$

(ii) Let $\mathcal{B}$ be the set of "bad" $t$ s satisfying $\left\langle 2(3 M)^{i} t\right\rangle \leq M^{-1 / 2}$ for some $i=1, \ldots, 3 l$. Then

$$
1-C l M^{-1 / 2}<\left|\sum_{j} \sigma_{j}(t)\right| \leq 1 \quad \forall t \notin \mathcal{B} .
$$

This follows because

$$
\sum_{j} \sigma_{j}(t)=2^{-l} \prod_{i=1}^{l}\left(s_{3 i-2}+s_{3 i-1}+s_{3 i}-s_{3 i-2} s_{3 i-1} s_{3 i}\right) .
$$

As explained above, $\left|r_{1}+r_{2}+r_{3}-r_{1} r_{2} r_{3}\right|=2$ and hence so does each term in the product (with an error of $C M^{-1 / 2}$ ) and the product has absolute value $2^{l}$. Further, an easy calculation shows that $x+y+z-x y z \leq 2$ for all $x, y, z \in[-1,1]$ so

$$
\left|\sum_{j} \sigma_{j}(t)\right| \leq 1 \quad \forall t
$$

both outside and inside $\mathcal{B}$. 
(iii) The spectra of $\sigma_{j}$ are disjoint. If $M>3 \operatorname{deg} f$ then they are also disjoint from spec $f$. This is also easy-in fact the spectra of any product of $s_{i}$ s are disjoint, and disjoint from spec $f$.

Let $\tau:=|f(0)|$, let $\varphi$ be defined by

$$
\varphi(t)= \begin{cases}\sqrt{\tau^{2}-|f(t)|^{2}} \frac{i f(t)}{|f(t)|}, & t \in[0, b], \\ 0, & \text { otherwise, }\end{cases}
$$

and let $P$ be a trigonometric polynomial approximating $\varphi,\|P-\varphi\|_{\infty}<\delta$. Assume $M>3 \operatorname{deg} P$ so that the spectra of $P \sigma_{j}$ are all disjoint and disjoint from spec $f$.

We are now in a position to define our first approximation step,

$$
\begin{aligned}
& f_{2}(t):=f(t)+\sum_{j=0}^{4^{l}-1} P \sigma_{j}(t), \\
& g_{2}(t):=g(t)+\sum_{j=0}^{4^{l}-1}\left(P \sigma_{j}\right)(t-j b) .
\end{aligned}
$$

It is clear that $\left|\widehat{f}_{2}(n)\right|=\left|\widehat{g}_{2}(n)\right|$ since the only difference between them is a rotation of each $P \sigma_{j}$. Since they have disjoint spectra, this preserves the absolute value of the Fourier transform.

Examine one $t \in[0, b] \backslash \mathcal{B}$. We use $(26)$ to sum over the $j$ s to get

$$
f_{2}=f \pm \varphi(t)+R(t), \quad|R| \leq C l M^{-1 / 2}+\delta \quad \forall t \in[0, b] \backslash \mathcal{B} .
$$

Notice that by Pythagoras' theorem [21], $|f \pm \varphi|=\tau$ for every $t \in[0, b]$, so

$$
|| f_{2}(t)|-\tau| \leq C l M^{-1 / 2}+\delta \quad \forall t \in[0, b] \backslash \mathcal{B} .
$$

On $[0, b] \cap \mathcal{B}$ we use 22] and 27 to get

$$
|f(t)|-\delta<\left|f_{2}(t)\right|<\tau+\delta .
$$

Finally, outside $[0, b]$ we have $\left|f_{2}(t)-f(t)\right| \leq \delta$ regardless of whether $t \in \mathcal{B}$ or not.

As for $g_{2}$, because the various translates $\varphi(t-j b)$ have disjoint supports we get (remember (25))

$$
\begin{array}{ll}
\left|g_{2}(t)-g(t)\right| \leq 2^{-l} \max \varphi+2^{l} \delta \leq C \sqrt{b / b^{\prime}}+2^{l} \delta & \forall t \in\left[0, b^{\prime}\right], \\
\left|g_{2}(t)-g(t)\right| \leq 2^{l} \delta & \forall t \notin\left[0, b^{\prime}\right] .
\end{array}
$$

These are the properties we need for $f_{2}$ and $g_{2}$.

Second approximation step. Now, $f_{2}$ and $g_{2}$ satisfy the conditions of the lemma except on the small set $\mathcal{B}$. On it we correct using Lemma 3 . Assume therefore that $\delta$ is sufficiently small and $M$ sufficiently large such that

$$
1-c_{1}<\left|f_{2}\right|<1+c_{1}
$$


and we can use Lemma 3 for $f_{2}$. Apply it with the parameter $\epsilon_{\text {Lemma } 3}=\frac{1}{2} \epsilon$ and the set

$$
E=\left\{t \in[0, b] \cap \mathcal{B}:\left|f_{2}(t)\right| \leq \tau-\frac{1}{2} \epsilon\right\} .
$$

If $\delta$ is sufficiently small and $M$ sufficiently large then by (28) we know that $E$ is contained in the interior of $\mathcal{B}$ and therefore the condition that $\left|f_{2}\right|$ is constant on $\partial E$ is satisfied. Call the resulting function $F$. We get

$$
\begin{aligned}
|| F(t)|-\tau| & \leq \epsilon \quad \forall t \in E, \\
\left|F(t)-f_{2}(t)\right| & \leq \frac{1}{2} \epsilon \quad \forall t \notin E .
\end{aligned}
$$

As for $\operatorname{deg} F$, since $\operatorname{deg} f_{2} \leq(3 M)^{3 l+1}$ we get

$$
\operatorname{deg} F \leq C(3 M)^{48 l+16} / \epsilon^{4} .
$$

This gives all properties required from $F$. Hence we need to define $G$. For this purpose examine the random function

$$
h:=\sum_{n} \pm \widehat{F-f_{2}}(n) e^{i n t}
$$

where the \pm stands for independent Bernoulli variables. It is well known (see [K85, Chapter 6, Theorem 2]) that with high probability

$$
\|h\|_{\infty} \leq C\left\|F-f_{2}\right\|_{2} \sqrt{\log \operatorname{deg} F}
$$

and in particular there exists a choice of signs $\xi_{n}$ satisfying this inequality. Define

$$
G:=g_{2}+\sum_{n} \xi_{n} \widehat{F-f_{2}}(n) e^{2 \pi i n t}
$$

Clearly we have $|\widehat{F}(n)|=|\widehat{G}(n)|$. Further, $\left\|F-f_{2}\right\|_{2} \leq C \sqrt{|\mathcal{B}|} \leq C l^{1 / 2} M^{-1 / 4}$ so

$$
\left\|G-g_{2}\right\|_{\infty} \leq C l^{1 / 2} M^{-1 / 4} \sqrt{l \log M+\log (1 / \epsilon)} .
$$

All that is required is to pick $\delta$ and $M$ correctly. Requirements (6) and (7) will follow if only $C^{-1 / 2}+\delta+\frac{1}{2} \epsilon<\epsilon$. To see $\sqrt{6}$ note that on $[0, b] \backslash \mathcal{B}$ it follows from 28, and (33). On $[0, b] \cap E$ it follows from $(32)$, and on $[0, b] \cap(\mathcal{B} \backslash E)$ from $(29)$, 33) and the definition of $E$. The proof of (7) is similar. Next, 23 will follow if the right hand side of 34) is $<\min \left\{\frac{1}{2} \epsilon, \sqrt{b / b^{\prime}}\right\}$ and $2^{l} \delta<\min \left\{\frac{1}{2} \epsilon, \sqrt{b / b^{\prime}}\right\}$ (remember 30 ). We remind the reader that in addition we assumed $M>3 \operatorname{deg} f, M>3 \operatorname{deg} P$, that

$$
\delta+C l M^{-1 / 2}<c_{1}-\max _{t}|| f(t)|-1|,
$$

which ensures 31 , and that $\delta+C l M^{-1 / 2}<\frac{1}{2} \epsilon$, which ensures that $\left|f_{2}\right|$ is constant on $\partial E$. Clearly choosing $\delta$ sufficiently small and then $M$ sufficiently large depending on $\delta$ (the most important dependency is via $M>3 \operatorname{deg} P$ ) will satisfy all these requirements and prove the lemma. 


\subsection{Intermediate remarks}

Since you reached so far down in the proof itself we believe you might be interested in some remarks on the structure of the proof more substantial than the ones given in the introduction. We start with a remark on Lemma 5. A tempting simplification is as follows: use Lemma 2 to correct $f$ to $F$ and then the probabilistic argument above to construct $G=g+\sum \pm \widehat{F-f}(n) e^{i n t}$. This would give the lemma with an additional benign-looking factor of $\sqrt{\log \operatorname{deg} F}$. However, this $\sqrt{\log }$ factor is not so easy to get rid of! To appreciate how serious a burden was removed, try to estimate the relation between $n=\operatorname{deg} f$ and $N=\operatorname{deg} F$ in Lemma 10 below. We got the tetration

$$
N \approx \underbrace{n^{n^{n}}}_{C \log ^{2}(1 / \epsilon) \text { times }}
$$

(this is after some optimizations; directly following the proof would give much more). This, by the way, is also the best we can say about the smoothness of the final $F$ and $G$, i.e. $\omega(\delta ; F)$ and $\omega(\delta ; G)$ decrease like an inverse tetration.

This is why we chose the current approach, and starting from Lemma 6 we no longer need to control the spectrum of $F$. Put differently, deg $F$ is the only parameter which gets worse when one increases the various parameters of our construction (e.g. the $l$ and $M$ of Lemma 5, the $l, N$ and $M$ of Lemma 7 below etc.). Removing the requirement to control $\operatorname{deg} F$ gives us the flexibility to increase these parameters with no punishment.

\subsection{The global correction scheme}

Lemma 6. Lemma 5 holds with $[a, b]$ replaced by a simple set $E$ if $[23]$ is replaced by

$$
\|g-G\|_{\infty} \leq C \sqrt{|E|} .
$$

Note that there is no $\left[a^{\prime}, b^{\prime}\right]$ in this formulation (or rather it is $[0,1]$ ).

Proof. Write $E$ as a disjoint union $E=I_{1} \cup \ldots \cup I_{N}$ and let $J_{1}, \ldots, J_{N}$ be disjoint intervals with $\left|J_{i}\right|=\left|I_{i}\right| /|E|$ (so they cover $[0,1]$ ). Denote by $\tau$ the common value of $|f(t)|$ for all $t \in \partial E$. Let $\epsilon_{2}$ be sufficiently small such that for any $\delta \leq \epsilon_{2}$ and any interval $I \subset E,\{t \in I:|f(t)|<\tau-\delta\}$ is an interval. Let $\epsilon_{3}=\min \left\{\epsilon, \epsilon_{2}, \sqrt{|E|}\right\}$. Now use Lemma 5 inductively $N$ times to get functions $f_{i}, g_{i}$ satisfying

(i) ||$f_{i}(t)|-\tau|<\epsilon_{3}\left(1-2^{-i-2}\right)$ for all $t \in \bigcup_{j=1}^{i} I_{j}$,

(ii) $\left|f_{i}(t)-f(t)\right| \leq \epsilon_{3}\left(\frac{1}{4}-2^{-i-2}\right)$ for all other $t \mathrm{~s}$,

(iii) $\left|g_{i}(t)-g(t)\right| \leq C \sqrt{|E|}+\epsilon_{3}\left(\frac{1}{4}-2^{-i-2}\right)$ for all $t \in \bigcup_{j=1}^{i} J_{j}$, and

(iv) $\left|g_{i}(t)-g(t)\right| \leq \epsilon_{3}\left(\frac{1}{4}-2^{-i-2}\right)$ for all other $t \mathrm{~s}$. 
Initiate the induction with $f_{0}=f, g_{0}=g$. We need to define the parameters for Lemma 5, most importantly the interval. Examine therefore $I_{i}$. Use the definition of $\epsilon_{2}$ with $\delta=\frac{3}{4} \epsilon_{3}$ to see that

$$
I_{i}^{*}:=\left\{t \in I_{i}:|f(t)|<\tau-\frac{3}{4} \epsilon_{3}\right\}
$$

is an interval. By the second induction assumption we know that $\left|f_{i-1}(t)\right|<\tau-\frac{1}{2} \epsilon_{3}$ for all $t \in I_{i}^{*}$ and $\left|f_{i-1}(t)\right|>\tau-\epsilon_{3}\left(1-2^{-i-1}\right)$ for all $t \in I_{i} \backslash I_{i}^{*}$. Let $I_{i}^{* *}$ be the component of $\left\{t \in I_{i}:\left|f_{i-1}(t)\right| \leq \tau-\frac{1}{2} \epsilon_{3}\right\}$ containing $I_{i}^{*}$. Now use Lemma 5 with the parameters as in the following table:

\begin{tabular}{|c||c|c|c|c|c||c|c|}
\hline Lemma 5 & $f$ & $g$ & $\epsilon$ & {$[a, b]$} & {$\left[a^{\prime}, b^{\prime}\right]$} & $F$ & $G$ \\
\hline here & $f_{i-1}$ & $g_{i-1}$ & $\epsilon_{3} 2^{-i-2}$ & $I_{i}^{* *}$ & $J_{i}$ & $f_{i}$ & $g_{i}$ \\
\hline
\end{tabular}

i.e. the lemma's output will be used to define $f_{i}$ and $g_{i}$. It is easy to verify (i) (iv) and the induction is complete. Now $F=f_{N}$ and $G=g_{N}$ are the desired functions.

Definition. Let $f$ be a trigonometric polynomial, let $E$ be a set and let $\max _{E}|f| \leq \alpha<$ $\beta$. Let $\epsilon>0$. Then an $\epsilon$-lifting of $f$ on $E$ from $\alpha$ to $\beta$ is a trigonometric polynomial $F$ extending $f$ such that

(i) $|f(t)-F(t)|<\epsilon$ for all $t \notin E$,

(ii) for all $t \in E$ minus a set of measure $<\epsilon$,

$$
(\beta-\alpha)+|f(t)|-\epsilon<|F(t)|<\beta+\epsilon,
$$

(iii) for all $t \in E$,

$$
|f(t)|-\epsilon<|F(t)|<\beta+\epsilon
$$

(iv) $\|F-f\|_{\infty}<2 \sqrt{\beta^{2}-\alpha^{2}}$.

See Figure 1. If $F$ and $G$ are two liftings of $f$ and $g$ which satisfy $|\widehat{F}(n)|=|\widehat{G}(n)|$ for all $n$ then we call them compatible liftings.

Lemma 7. Let $f$ and $g$ be as in Lemma 5. Let $\epsilon>0$ be some parameter. Let $l \geq 0$ be some integer, let I be an interval with length $\leq 4^{-l}$ and let $J$ be an interval of length $4^{l}|I|$. Assume that $|f(t)|<\tau$ for all $t \in I$ where $\tau<1$. Then there exist compatible $\epsilon$-liftings $F$ of $f$ on I from $\tau$ to 1 and $G$ of $g$ on $J$ from any $v \geq \max _{J}|g|$ to $\mu=\sqrt{v^{2}+4^{-l}\left(1-\tau^{2}\right)}$.

Proof. We may assume $I=[0, b]$ and $J=\left[0,4^{l} b\right]$. Define two parameters $N$ and $M$ that will accompany us throughout the proof and will be fixed at the end (think of both as large but of $M$ as being much larger than $N$ ). The proof is very similar to the proof of Lemma 5, and in particular we retain the notations $\psi$ and $\psi_{[\mathrm{m}]}$. We shall repeat the construction of the $\sigma_{j}$ s of Lemma 5, $N$ times in disjoint spectra. Namely, we define functions $\sigma_{j}^{q}, j=0, \ldots, 4^{l}-1$ and $q=0, \ldots, N-1$, satisfying 


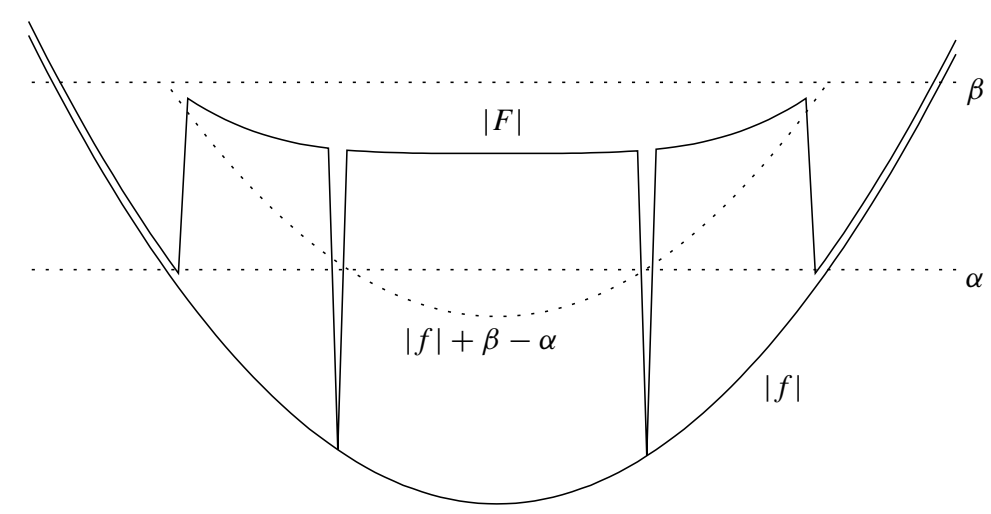

Fig. 1. $F$ is a lifting of $f$ from $\alpha$ to $\beta$ on the set $E$ where $|f| \leq \alpha$. The graph depicts only the absolute values of $f$ and $F .|F|$ is drawn here taking the value $\sqrt{|f|^{2}+\beta^{2}-\alpha^{2}}$ which is what will typically happen in our construction.

(i) Let $\mathcal{B}$ be the set of "bad" $t$ s satisfying $\left\langle 2(3 M)^{i} t\right\rangle \leq M^{-1 / 2}$ for some $i=1, \ldots, 3 l N$. Then

$$
2^{-l}-C l M^{-1 / 2}<\left|\sigma_{j}^{q}(t)\right| \leq 2^{-l} \quad \forall j, q, t \notin \mathcal{B} .
$$

Further, $\left|\sigma_{j}^{q}(t)\right| \leq 2^{-l}$ for all $t$.

(ii) For any $t$ and $q,\left|\sum_{j} \sigma_{j}^{q}(t)\right| \leq 1$ and further

$$
1-C l M^{-1 / 2}<\left|\sum_{j} \sigma_{j}^{q}(t)\right| \leq 1 \quad \forall q, t \notin \mathcal{B} .
$$

(iii) The spectra of $P \sigma_{j}^{q}$ are disjoint. Here $P$ is any polynomial with $\operatorname{deg} P \leq M$.

Let $P$ be an $M$-approximation of the indicator function $\mathbf{1}_{[0, b / N]}$ (in the same sense of $\psi$ in Lemma 5) so that $P$ is real, $|P| \leq 1$ and

$$
\left|P(t)-\mathbf{1}_{[0, b / N]}(t)\right| \leq C M^{-1 / 2}+C \frac{N / b}{M} \quad \forall t, d(t,\{0, b / N\})>M^{-1 / 2},
$$

where $d$ is the usual distance of a point from a set considered periodically. We will assume henceforth that $M>N^{2} b^{-2}$ and avoid carrying the $N / b M$ term. This allows us to define our functions

$$
\begin{aligned}
& F=f+\sqrt{1-\tau^{2}} \sum_{q=0}^{N-1} \sum_{j=0}^{4^{l}-1} \frac{i f(q b / N)}{|f(q b / N)|}\left(P \sigma_{j}^{q}\right)(t-q b / N), \\
& G=g+\sqrt{1-\tau^{2}} \sum_{q=0}^{N-1} \sum_{j=0}^{4^{l}-1} \frac{i g(j b+q b / N)}{|g(j b+q b / N)|}\left(P \sigma_{j}^{q}\right)(t-(j b+q b / N)) .
\end{aligned}
$$


As in Lemma 5 it is clear that $|\widehat{F}(n)|=|\widehat{G}(n)|$ since each term is rotated and multiplied by a unimodular number. Define

$$
\mathcal{B}^{\prime}:=\left(\bigcup_{q=0}^{N-1} \bigcup_{j=0}^{4^{l}-1}\left(\mathcal{B} \cup\left[-M^{-1 / 2}, M^{-1 / 2}\right]\right)+j b+q b / N\right)
$$

where the + here stands for usual set addition, i.e. $B+x:=\{b+x: b \in B\}$. Examine some $t \notin \mathcal{B}^{\prime}$. We use (37) to sum over the $j$ s in 39] to get

$$
F=f \pm \sqrt{1-\tau^{2}} \sum_{q=0}^{N-1} \frac{i f(q b / N)}{|f(q b / N)|} P(t-q b / N)+O\left(l N M^{-1 / 2}\right) .
$$

By (38) all the terms in the sum over $q$ give $P=O\left(M^{-1 / 2}\right)$ unless $t \in[0, b]$, in which case the term $q=\lfloor N t / b\rfloor$ gives $P=1+O\left(M^{-1 / 2}\right)$. We get

$$
F(t)=f(t) \pm \sqrt{1-\tau^{2}} \frac{i f(q b / N)}{|f(q b / N)|}+O\left(l N M^{-1 / 2}\right) \quad \forall t \in[0, b] \backslash \mathcal{B}^{\prime} .
$$

Now we use Pythagoras' theorem (21) for $\eta=\tau f(q b / N) /|f(q b / N)|$ to get

$$
\begin{aligned}
\mid 1 & -\left|f(q b / N) \pm \sqrt{1-|\eta|^{2}} \frac{i \eta}{|\eta|}\right| \mid \\
& \leq\left|\left(\eta \pm \sqrt{1-|\eta|^{2}} \frac{i \eta}{|\eta|}\right)-\left(f(q b / N) \pm \sqrt{1-|\eta|^{2}} \frac{i \eta}{|\eta|}\right)\right|=\tau-|f(q b / N)|,
\end{aligned}
$$

which allows us to estimate, for any $t \in[0, b] \backslash \mathcal{B}^{\prime}$,

$$
\begin{aligned}
|F(t)| & \stackrel{42 \mid}{\geq}\left|f\left(\frac{q b}{N}\right) \pm \sqrt{1-|\eta|^{2}} \frac{i \eta}{|\eta|}\right|-\left|f(t)-f\left(\frac{q b}{N}\right)\right|-C_{N} M^{-1 / 2} \\
& \stackrel{\text { 433 }}{\geq} 1-\tau+\left|f\left(\frac{q b}{N}\right)\right|-\left|f(t)-f\left(\frac{q b}{N}\right)\right|-C_{N} M^{-1 / 2} \\
& \geq 1-\tau+|f(t)|-2 \omega(b / N ; f)-C_{N} M^{-1 / 2} .
\end{aligned}
$$

Set $\omega:=2 \omega(b / N ; f)+C l N M^{-1 / 2}$. In the other direction, 22) gives $|f(q b / N)| \leq$ $\left|f(q b / N)+\sigma(i \eta /|\eta|) \sqrt{1-\tau^{2}}\right| \leq 1$ for any $\sigma \in[-1,1]$. We take the variation in $f$ into consideration as above and get

$$
\begin{aligned}
(1-\tau)+|f(t)|-\omega & \leq|F(t)| \leq 1+\omega & \forall t \in[0, b] \backslash \mathcal{B}^{\prime}, \\
|f(t)|-\omega \leq|F(t)| & \leq 1+\omega & \forall t \in[0, b] \cap \mathcal{B}^{\prime} .
\end{aligned}
$$

Finally, we also have $\|F-f\|_{\infty} \leq \sqrt{1-\tau^{2}}+\omega$.

Next we move to examine $G$. This time we first notice that the only meaningful term in (40) is the one for which $j=\lfloor t / b\rfloor$ and $q=\lfloor N(t / b-j)\rfloor$. So we have (again for $\left.t \notin \mathcal{B}^{\prime}\right)$

$$
G(t)=g(t)+\sqrt{1-\tau^{2}} \frac{i g(t+R(t))}{|g(t+R(t))|} \sigma_{\lfloor t / b\rfloor}(R(t))+O\left(2^{l} N M^{-1 / 2}\right), \quad|R(t)| \leq b / N
$$


(we get here a $2^{l}$ factor in the error, compared to the $l$ in 41 because the $\sigma_{j} \mathrm{~s}$ are no longer "synchronized" so we cannot use (37) and have to use (36) and sum over all the terms). We now use (36) to get

$$
G(t)=g(t) \pm 2^{-l} \sqrt{1-\tau^{2}} \frac{i g(t+R(t))}{|g(t+R(t))|}+O\left(2^{l} N M^{-1 / 2}\right), \quad \forall t \in\left[0, b^{\prime}\right] \backslash \mathcal{B}^{\prime} .
$$

A calculation similar to the one done with $f$ shows that

$$
\begin{gathered}
\mu-v+|g(t)|-\omega^{\prime} \leq|G(t)| \leq \mu+\omega^{\prime} \quad \forall t \in\left[0, b^{\prime}\right] \backslash \mathcal{B}^{\prime}, \\
|g(t)|-\omega^{\prime} \leq|G(t)| \leq \mu+\omega^{\prime} \quad \forall t \in\left[0, b^{\prime}\right] \cap \mathcal{B}^{\prime}, \\
\omega^{\prime}:=2 \omega(b / N ; g)+C 2^{l} N M^{-1 / 2}
\end{gathered}
$$

and $\|G-g\|_{\infty} \leq \sqrt{1-\tau^{2}}+\omega^{\prime}$.

With these estimates the lemma will be finished once we pick $N$ and $M$. First pick $N$ such that $\omega(b / N ; f)<\min \left\{\frac{1}{4} \epsilon, \frac{1}{4} \sqrt{1-\tau^{2}}\right\}$ and similarly for $g$. Next pick $M$ to satisfy all past requirements. They are all of the type " $M$ is sufficiently large (possibly depending on $N, l$ and $\epsilon$ )". Here is the full list (in chronological order): $M>N^{2} b^{-2}, M>3 \operatorname{deg} f$, $C l N M^{-1 / 2}<\min \left\{\frac{1}{2} \epsilon, \frac{1}{2} \sqrt{1-\tau^{2}}\right\}$ (which bounds $\omega$ and ensures $|F(t)-f(t)|<\epsilon$ outside $[0, b])$ and $C 4^{l} N M^{-1 / 2}<\epsilon$, which ensures $\left|\mathcal{B}^{\prime}\right|<\epsilon, \omega^{\prime}<\epsilon$ and $|G(t)-g(t)|<$ $\epsilon$ outside $\left[0, b^{\prime}\right]$. With all these satisfied we get everything we want for $F$ and $G$.

Again we need to generate a set version from the interval version. We trust that by now the reader will have no problem to prove:

Lemma 8. Let $f, g, \epsilon, \tau$ and $l$ be as in Lemma 7. Let $E$ be a simple set with $|E|=4^{-l}$ such that $|f(t)|<\tau$ for all $t \in E$. Then one can find compatible $\epsilon$-liftings $F$ of $f$ on $E$ from $\tau$ to 1 and $G$ of $g$ on $[0,1]$ from $v \geq\|g\|_{\infty}$ to $\mu=\sqrt{v^{2}+4^{-l}\left(1-\tau^{2}\right)}$.

In the next lemma we get rid of the errors in the exceptional sets (compare clauses (i) and (iii) below to requirement (35) from the definition of lifting). Hence it will be convenient to use the following definition: The oscillation of the absolute value of a function $g$ is

$$
\operatorname{Osc}(g):=\max _{t}|g(t)|-\min _{t}|g(t)| \text {. }
$$

Lemma 9. Let $f$ and $g$ be as in Lemma 5 but with the additional requirements

$$
1-c_{2}<|f|<1, \quad 1-c_{2}<|g|<1+c_{2}
$$

for some absolute constant $c_{2}>0$. Let $\epsilon>0$ be some parameter. Then one can extend $f$ and $g$ to $F$ and $G$ such that

(i) $1-\frac{1}{2}\|1-|f|\|_{\infty}-\epsilon<|F(t)|<1+\epsilon$ for all $t$,

(ii) $\|F-f\|_{\infty}<C \sqrt{\|1-|f|\|_{\infty}}$,

(iii) $\operatorname{Osc}(G)<\operatorname{Osc}(g)+\epsilon$,

(iv) $\|G-g\|_{\infty}<C \sqrt{\|1-|f|\|_{\infty}}$. 
Proof. Set $\tau=1-\frac{1}{2}\|1-|f|\|_{\infty}$. Let

$$
E=\left\{t:|f(t)|<\tau-\frac{1}{4} \epsilon\right\}
$$

and write $|E|$ in base 4 ,

$$
|E|=\sum_{l=1}^{\infty} \alpha_{l} 4^{-l}, \quad \alpha_{l} \in\{0,1,2,3\} .
$$

Divide $E$ into simple sets $E_{l, i}, l=1,2, \ldots$ and $i=0, \ldots, \alpha_{l}-1$, with $\left|E_{l, i}\right|=4^{-l}$. Order these sets by increasing $l$ and call the resulting sequence $\left\{E_{j}\right\}_{j=1}^{\infty}$. We now create two sequences of polynomials $f_{i}$ and $g_{i}$ with $\left|\widehat{f}_{i}(n)\right|=\left|\widehat{g}_{i}(n)\right|$ for all $n$ and all $i$ inductively by using Lemma 8 (at the $i$ th step) with $f_{i-1}, g_{i-1}$, the set $E_{i}$, the parameters $\epsilon_{\mathrm{Lemma}} 8=\delta 2^{-i}$ ( $\delta$ is some parameter $<\frac{1}{7} \epsilon$ to be fixed later) and $\tau$ and with $v_{\text {Lemma } 8}=\mu_{i-1}+\delta 2^{-i}$ (the induction is initialized with $f_{0}=f, g_{0}=g$ and $\left.\mu_{0}=\|g\|_{\infty}\right)$. Call the output of the lemma $f_{i}, g_{i}$ and $\mu_{i}$. It is easy to verify that

(i) $\tau-\frac{1}{7} \epsilon<\left|f_{i}(t)\right|<1+\frac{1}{7} \epsilon$ except on a set $\mathcal{B}$ of measure $\delta+4^{1-i / 4}$ (the $\delta$ error is the combined error from the previous stages while $4^{1-i / 4}$ is the set of $E_{j}$ s not yet handled).

(ii) Uniformly we have

$$
\left\|f_{i}-f\right\|_{\infty}<2 \sqrt{1-\tau^{2}}+\delta .
$$

This requires both (i) and (iv) from the definition of lifting.

(iii) Outside a set of measure $<\delta$ we have

$$
\begin{aligned}
\left|g_{i}(t)\right| & >\left|g_{i-1}(t)\right|+\mu_{i}-\left(\mu_{i-1}+\delta 2^{-i}\right)-\delta 2^{-i} \\
& >\left|g_{i-2}(t)\right|+\mu_{i}-\mu_{i-2}-2 \delta\left(2^{1-i}+2^{-i}\right)>\cdots \\
& >|g(t)|+\mu_{i}-\|g\|_{\infty}-2 \delta .
\end{aligned}
$$

We prefer to write this as

$$
\mu_{i}-\operatorname{Osc}(g)-\frac{2}{7} \epsilon<\left|g_{i}(t)\right|<\mu_{i}+\frac{1}{7} \epsilon .
$$

(iv) Uniformly

$$
\left\|g_{i}-g\right\|_{\infty}<2 \sqrt{1-\tau^{2}}+\delta .
$$

Take some $i$ sufficiently large (to be fixed later) and examine $f_{i}$ and $g_{i}$.

We now correct over the exceptional sets using Lemma 6 twice. First use the lemma with $f_{i}, g_{i}$, the set $\mathcal{B}:=\left\{t:\left|f_{i}(t)\right| \leq \tau-\frac{1}{7} \epsilon\right\}$ and $\epsilon_{\text {Lemma } 6}=\frac{1}{7} \epsilon$. To enable this, fix

$$
c_{2}:=\min \left\{\frac{1}{4} c_{1}, 1-\sqrt{1-c_{1}^{2} / 64}\right\} .
$$

With this value, the fact that $\tau>1-c_{2}$ implies that $2 \sqrt{1-\tau^{2}}<\frac{1}{4} c_{1}$ and hence for $\delta<\frac{1}{4} c_{1}$, 45 and 44 give $1-c_{1}<\left|f_{i}\right|<1+c_{1}$ and Lemma 6 may indeed be applied. 
Call the resulting functions $F^{*}$ and $G^{*}$. Since $|\mathcal{B}|<\delta+4^{1-i / 4}$ it is clear that for $\delta$ sufficiently small and $i$ sufficiently large we get

$$
\left\|g_{i}-G^{*}\right\|_{\infty}<\min \left\{\frac{1}{7} \epsilon, \frac{1}{4} c_{1}\right\} .
$$

This corrects $F^{*}$ in the sense that now

$$
\tau-\frac{2}{7} \epsilon<\left|F^{*}(t)\right|<1+\frac{2}{7} \epsilon \text { for all } t .
$$

Now use Lemma 6 with $f_{\text {Lemma }}=G^{*}, g_{\text {Lemma }}=F^{*}$ and the set $\mathcal{B}^{*}:=\{t$ : $\left.\left|G^{*}(t)\right| \leq \mu_{i}-\operatorname{Osc}(g)-\frac{3}{7} \epsilon\right\}$ and again $\epsilon_{\text {Lemma } 6}=\frac{1}{7} \epsilon$. The resulting functions are our $G$ and $F$. As above, from the definition (48) of $c_{2}$, 44, 47) and 49, we get $1-c_{1}<$ $\left|G^{*}\right|<1+c_{1}$ so if $\delta<\frac{1}{4} c_{1}$ we may apply the lemma. Further,

$$
\left|\mathcal{B}^{*}\right| \stackrel{49}{\leq}\left|\left\{t:\left|g_{i}\right| \leq \mu_{i}-\operatorname{Osc}(g)-\frac{2}{7} \epsilon\right\}\right| \stackrel{46}{<} \delta
$$

so that $\left\|F-F^{*}\right\|_{\infty} \leq C \sqrt{\delta}$ and if $\delta$ is sufficiently small, $\left\|F-F^{*}\right\|_{\infty} \leq \frac{1}{7} \epsilon$ so with 50 , we have what we need for $F$. For $G$ we get

$$
\mu_{i}-\operatorname{Osc}(g)-\frac{4}{7} \epsilon<|G(t)|<\mu_{i}+\frac{3}{7} \epsilon
$$

$\operatorname{so} \operatorname{Osc}(G)<\operatorname{Osc}(g)+\epsilon$. Fixing $\delta$ sufficiently small and $i$ sufficiently large to satisfy all the past requirements completes the proof of the lemma.

Lemma 10. Let $f, g$ and $\epsilon$ be as in Lemma 5 but with the additional requirement

$$
1-c_{3}<|f|<1, \quad 1-c_{3}<|g|<1+c_{3} .
$$

Then one can extend $f$ and $g$ to $F$ and $G$ such that

(i) $1-\epsilon<|F(t)|<1+\epsilon$ for all $t$,

(ii) $\|F-f\|_{\infty}<C \sqrt{\|1-|f|\|_{\infty}}$,

(iii) $\operatorname{Osc}(G)<\operatorname{Osc}(g)+\epsilon$,

(iv) $\|G-g\|_{\infty}<C \sqrt{\|1-|f|\|_{\infty}}$.

Proof. This follows easily by applying Lemma 9 repeatedly (to preserve the requirement $|f|<1$ you need to multiply $f$ and $g$ by normalization factors). Let us do it in detail nonetheless. Write $\rho=\|1-|f|\|_{\infty}$ and let $\delta$ be some parameter sufficiently small to be fixed later. We define $f_{0}=f$ and $g_{0}=g$ and then inductively $f_{i}$ and $g_{i}$ with the following properties:

(a) $\left|\widehat{f}_{i}(n)\right|=\left|\widehat{g}_{i}(n)\right|$ for all $n,\left(1+\delta 2^{-i}\right) f_{i}$ extends $f_{i-1}$ and $\left(1+\delta 2^{-i}\right) g_{i}$ extends $g_{i-1}$.

(b) $1-(\rho+2 i \delta) 2^{-i}<\left|f_{i}\right|<1$.

(c) $\left\|f_{i}-f_{i-1}\right\|_{\infty} \leq C \sqrt{\rho 2^{-i}}$ for $i>0$.

(d) $\operatorname{Osc}\left(g_{i}\right)<\operatorname{Osc}(g)+\delta\left(1-2^{-i}\right)$.

(e) $\left\|g_{i}-g_{i-1}\right\|_{\infty} \leq C \sqrt{\rho 2^{-i}}$ for $i>0$. 
Let us verify the induction steps. At the $i$ th step $(i \geq 1)$ we wish to apply Lemma 9 to the functions $f_{i-1}$ and $g_{i-1}$. For this to hold we must know that (44) holds for our functions $f_{i-1}$ and $g_{i-1}$. First we note that if $c_{3}<c_{2}$ and $\delta<\frac{1}{3} c_{2}$ then the second induction assumption ensures that $1-c_{2}<\left|f_{i-1}\right|<1$. As for $g_{i-1}$, we first estimate $\left\|g_{i-1}\right\|_{\infty}$ by noting that

$$
\begin{gathered}
\left\|g_{i-1}\right\|_{\infty} \leq\left\|g_{i-1}\right\|_{2}+\operatorname{Osc}\left(g_{i-1}\right) \stackrel{\text { (d) }}{\leq}\left\|g_{i-1}\right\|_{2}+\operatorname{Osc}(g)+\delta \\
\stackrel{(a)]}{=}\left\|f_{i-1}\right\|_{2}+\operatorname{Osc}(g)+\delta \stackrel{(b)}{\leq} 1+2 c_{3}+\delta .
\end{gathered}
$$

A similar calculation shows that

$$
\left\|g_{i-1}\right\|_{\infty} \geq 1-(\rho+2 i \delta) 2^{-i}-2 c_{3}-\delta \geq 1-3 c_{3}-2 \delta .
$$

With these estimates we write

$$
\left\|\left|g_{i-1}\right|-1\right\|_{\infty} \leq \operatorname{Osc}\left(g_{i-1}\right)+\left|\left\|g_{i-1}\right\|_{\infty}-1\right| \leq 5 c_{3}+3 \delta .
$$

Hence if we define $c_{3}:=\frac{1}{10} c_{2}$ and ensure $\delta<\frac{1}{6} c_{2}$ then the requirements for Lemma 9 are ensured.

We now apply Lemma 9 with $\epsilon_{\text {Lemma } 9}=\delta 2^{-i}$. Call the resulting functions $f^{*}$ and $g^{*}$ and define $f_{i}=f^{*} /\left(1+\delta 2^{-i}\right)$ and $g_{i}=g^{*} /\left(1+\delta 2^{-i}\right)$. It is quite easy to verify that all the inductive assumptions hold-let us do two examples in detail. First, let us verify the left hand inequality of (b) We have

$$
\left|f_{i}\right| \geq \frac{1-\frac{1}{2}\left\|1-\left|f_{i-1}\right|\right\|-\delta 2^{-i}}{1+\delta 2^{-i}} \stackrel{(\mathrm{b})}{>} \frac{1-(\rho+(2 i-1) \delta) 2^{-i}}{1+\delta 2^{-i}}>1-(\rho+2 i \delta) 2^{-i}
$$

where the inequality labeled (b) uses this clause for $i-1$ inductively. Secondly we discuss (d) We have $\operatorname{Osc}\left(g_{i}\right)=\operatorname{Osc}\left(g^{*}\right) /\left(1+\delta 2^{-i}\right)<\operatorname{Osc}\left(g^{*}\right)$ while clause (iii) of Lemma 9 gives $\operatorname{Osc}\left(g^{*}\right)<\operatorname{Osc}\left(g_{i-1}\right)+\delta 2^{-i}$. This completes the induction.

Now take $i$ sufficiently large and define $\lambda_{i}=\prod_{j=1}^{i}\left(1+\delta 2^{-j}\right), F:=f_{i} \lambda_{i}$ and $G:=g_{i} \lambda_{i}$. We note that

$$
|F| \leq \lambda_{i} \leq 1+C \delta, \quad|F| \geq 1-(\rho+2 i) 2^{-i} \geq 1-C 2^{-i},
$$

hence for $i$ sufficiently large and $\delta$ sufficiently small the first requirement on $F$ is satisfied. The other requirements on $F$ and $G$ may be verified with similar ease.

Proof of the Theorem. Let $\varphi: \mathbb{T} \rightarrow \mathbb{T}$ be a function which makes one rotation around 0 very quickly, namely for some $\epsilon>0$ to be fixed later define

$$
\varphi(t)= \begin{cases}e^{2 \pi i t / \epsilon}, & t \leq \epsilon \\ 1, & t>\epsilon\end{cases}
$$


Let $f_{1}$ be a trigonometric polynomial satisfying $\left\|f_{1}-\varphi\right\|_{\infty}<\epsilon, \operatorname{deg} f_{1}<C \epsilon^{-C}$. Clearly wind $f_{1}=1$ and $\left\|f_{1}-1\right\|_{2}<C \sqrt{\epsilon}$. Examine the random function

$$
h=\sum_{n \neq 0} \pm \widehat{f_{1}(n)} e^{i n t}
$$

As before we have $\left.\|h\|_{\infty} \leq C \sqrt{\epsilon \log (1 / \epsilon}\right)$ with high probability. Pick one combination of signs $\xi_{n}$ such that this holds and define $g_{1}=\widehat{f_{1}}(0)+\sum \xi_{n} \widehat{f_{1}}(n) e^{i n t}$. Clearly $\left|\widehat{f_{1}}(n)\right|=$ $\left|\widehat{g_{1}}(n)\right|$ and if $\epsilon$ is sufficiently small, wind $g_{1}=0$.

We now apply Lemma 10 inductively as follows. For even $j$ s we apply it with

$$
f_{\text {Lemma } 10]}=f_{j-1} v_{j}, \quad g_{\text {Lemma } 10}=g_{j-1} v_{j}, \quad v_{j}=\frac{1}{\max \left\{\left\|f_{j-1}\right\|_{\infty}, 1\right\}},
$$

and then define $f_{j}=F_{\text {Lemma } 10}$ and $g_{j}=G_{\text {Lemma[10. }}$. For odd $j$ s we reverse the roles of $f$ and $g$, i.e. take the normalization factor to be $v_{j}=1 / \max \left\{\left\|g_{j-1}\right\|_{\infty}, 1\right\}$ and then $f_{\text {Lemma } 10]}=v_{j} g_{j-1}$ and $g_{\text {Lemma } 10}=v_{j} f_{j-1}$ etc. In both cases we take $\epsilon_{\text {Lemma } 10]}=2^{-j} \epsilon$. An argument similar to that of Lemma 10 now shows that throughout this process

$$
\operatorname{Osc}\left(f_{j}\right)<5 \epsilon 2^{-j}, \quad \operatorname{Osc}\left(g_{j}\right)<5 \epsilon 2^{-j} .
$$

Let us show (51) for the case of $j$ even (the other case is identical). Here $f_{j}$ follows immediately since by clause (i) of Lemma $10, \operatorname{Osc}\left(f_{j}\right)<2 \epsilon 2^{-j}$. As for $g$, the same clause (i) in step $j-1$ shows that $\operatorname{Osc}\left(g_{j-1}\right)<4 \epsilon 2^{-j}$ so $\operatorname{Osc}\left(g_{j-1} v_{j}\right)<4 \epsilon 2^{-j}$. We apply clause (iii) of Lemma 10 in step $j$ and get $\operatorname{Osc}\left(g_{j}\right)<5 \epsilon 2^{-j}$.

A similar argument shows that both are close to 1 in the sense that

$$
\left\|\left|f_{j}\right|-1\right\|_{\infty}<6 \epsilon 2^{-j}, \quad\left\|\left|g_{j}\right|-1\right\|_{\infty}<6 \epsilon 2^{-j} .
$$

Again we demonstrate this under the assumption that $j$ is even. For $f_{j}$ this is immediate from clause (i) of Lemma 10 For $g_{j}$, since $\left\|g_{j}\right\|_{2}=\left\|f_{j}\right\|_{2}$ we see that $\left|\left\|g_{j}\right\|_{2}-1\right|<\epsilon 2^{-j}$ and since $\left|g_{j}(t)-\left\|g_{j}\right\|_{2}\right| \leq \operatorname{Osc}\left(g_{j}\right)$ we get (52).

This implies that if $\epsilon$ is sufficiently small the induction actually works in the sense that $1-c_{3}<v_{j} f_{j-1}<1+c_{3}$ and $1-c_{3}<v_{j} g_{j-1}<1+c_{3}$ are preserved throughout. Further it implies $\left\|f_{j+1}-f_{j}\right\|_{\infty}<C \sqrt{\epsilon 2^{-j}}$ and in particular we find that (if $\epsilon$ is sufficiently small) wind $f_{j}=$ wind $f_{j+1}, f=\lim f_{j}$ exists and is continuous, $|f(t)|=1$ for all $t$ and wind $f=$ wind $f_{1}=1$. Similarly we deduce that $g=\lim g_{j}$ exists and is continuous, $|g(t)|=1$ for all $t$ and wind $g=0$. The property that $\left|\widehat{f}_{j}(n)\right|=\left|\widehat{g}_{j}(n)\right|$ is preserved in the limit so $|\widehat{f}(j)|=|\widehat{g}(j)|$ for all $j$ and the theorem is proved.

\section{References}

[BGP91] Boutet de Monvel-Berthier, A., Georgescu, V., Purice, R.: A boundary value problem related to the Ginzburg-Landau model. Comm. Math. Phys. 142, 1-23 (1991) Zbl 0742.35045 MR 1137773 
[B06] Brezis, H.: New questions related to the topological degree. In: The Unity of Mathematics, Progr. Math. 244, Birkhäuser Boston, Boston, MA, 137-154 (2006) Zbl 05050075 MR 2181804

[BC83] Brezis, H., Coron, J.-M.: Large solutions for harmonic maps in two dimensions. Comm. Math. Phys. 92, 203-215 (1983) Zbl 0532.58006 MR 0728866

[BN95] Brezis, H., Nirenberg, L.: Degree theory and BMO. I. Compact manifolds without boundaries. Selecta Math. (N.S.) 1, 197-263 (1995) Zbl 0852.58010 MR 1354598

[J99] Jaming, P.: Phase retrieval techniques for radar ambiguity problems. J. Fourier Anal. Appl. 5, 309-329 (1999) Zbl 0940.94003 MR 1700086

[K85] Kahane, J.-P.: Some Random Series of Functions. 2nd ed., Cambridge Stud. Adv. Math. 5, Cambridge Univ. Press, Cambridge (1985) Zbl 0571.60002 MR 0833073

[K05] Kahane, J.-P.: Sur l'équation fonctionnelle $\int_{\mathrm{T}}(\psi(t+s)-\psi(s))^{3} d s=\sin t$. C. R. Math. Acad. Sci. Paris 341, 141-145 (2005) Zbl pre02210371 MR 2158834

[K99] Korevaar, J.: On a question of Brézis and Nirenberg concerning the degree of circle maps. Selecta Math. (N.S.) 5, 107-122 (1999) Zbl 0938.55005 MR 1694896

[KJF77] Kufner, A., John, O., Fučík, S.: Function Spaces. Noordhoff, Leyden, and Academia, Prague (1977) Zbl 0364.46022 MR 0482102

[dLKK77] de Leeuw, K., Katznelson, Y., Kahane, J.-P.: Sur les coefficients de Fourier des fonctions continues. C. R. Acad. Sci. Paris Sér. A-B 285, A1001-A1003 (1977) Zbl 0372.42004 MR 0510870

[Z68] Zygmund, A.: Trigonometric Series. Vols. I, II, 2nd ed., Cambridge Univ. Press, London (1968) MR 0236587 